\title{
Owners' perception of carboplatin in conjunction with other palliative treatments for cancer therapy
}

\author{
D. B. Bowles, M. C. Robson, P. E. Galloway and L. Walker
}

OBJECTIVES: To determine owners' perception of their pet's quality of life during treatment with carboplatin for a variety of canine and feline neoplasms. METHODS: Owners were contacted via a postal questionnaire and asked questions regarding their perception of chemotherapy in pets and their perception of carboplatin treatment in their pet. RESULTS: Twenty-eight (59\%) of owners responded to the questionnaire. Forty-three percent of owners had not considered chemotherapy in pets before treatment; however, after treatment, 89\% of owners supported its use. Sixteen (57\%) patients had mild to severe side effects. Most patients experienced mild side effects, including lethargy and loss of appetite. Quality of life during treatment was reduced compared with prediagnosis quality of life however at its best was significantly improved compared with pretreatment quality of life. Eighty-nine per cent of respondents did not regret treating their pet. CLINICAL SIGNIFICANCE: Carboplatin is well tolerated by both owners and pets. Most patients have either no side effects or experience mild lethargy or inappetence. Carboplatin treatment, either alone or in conjunction with other medications, should be considered as a palliative treatment in both dogs and cats with susceptible neoplasms.

Carboplatin (cis-diammine-1,1-cyclobutane decarboxylate platinum (II) carboplatin) is a second generation platinum chemotherapy agent that is used in a variety of tumours in dogs and cats (Page and others 1993, Kisseberth and others 2008). Phase I clinical trials have been performed in both dogs and cats, and a maximum toxic dose and suggested dosing schedule have been established in both species (Page and others 1993, Kisseberth and others 2008). Reported dosing regimes are $300 \mathrm{mg} / \mathrm{m}^{2}$ intravenously every 3 weeks in dogs, and 200 to $240 \mathrm{mg} / \mathrm{m}^{2}$ intravenously every 28 days in cats (Hahn and others 1997, Kisseberth and others 2008).

The side effect profile has also been documented in both species. Carboplatin appears to be well tolerated with mild to moderate gastrointestinal and hematologic side effects (neutropenia and thrombocytopenia) being most common (Page and others 1993, Hahn and others 1997, Kisseberth and others 2008).

Myelosuppression is the dose-limiting toxicity in both dogs and cats (Page and others 1993, Kisseberth and others 2008). In dogs, the reported nadir for both neutrophils and platelets is around 14 days, with recovery by 21 days after treatment. In cats, the nadir of neutrophils is seen between 17 and 21 days, with recovery evident in most patients by day 28 after treatment (Fox 2000). Thrombocytopenia is seen infrequently in cats; however, the nadir is similar to the neutrophil nadir (Kisseberth and others 2008). For most veterinary patients, carboplatin is a palliative treatment. Reported uses for carboplatin include as a single agent or part of a multi-modality treatment protocol for various carcinomas (Murphy and others 2006, Dominquez and others 2009), as part of a treatment regime for canine nasal tumours (Langova and others 2004) and anal sac adenocarcinomas (Bennett and others 2002) and as an adjunctive treatment for canine appendicular osteosarcoma (Bergman and others 1996) and malignant melanoma (Freeman and others 2003). Intravenous carboplatin use in cats is less frequently reported; however, reported uses include extraskeletal osteosarcoma (Spugnini and others 2001, Dhaliwal and others 2003) and a variety of sarcomas and carcinomas (Kisseberth and others 2008). Carboplatin has also been reported to be administered by intracavitary injection for mesothelioma in dogs and cats (Spugnini and others 2008) and intralesional injection for feline squamous cell carcinoma (Theon and others 1996).

Evaluation of veterinary patient quality of life is a relatively recent concept (Yeates and Main 2009). Many human oncology studies focus on evaluating patient quality of life as well as more traditional survival parameters (Osoba 1992). In contrast, veterinary studies have historically focussed on survival parameters, such as median survival time, time to tumour progression and quantitative assessment of tolerability and side effects. Although owners are interested in this information, many owners are very concerned about their pet's quality of life during the treatment, especially, if the treatment is palliative (Mellanby and others 2003). One study involving cardiac patients showed that the patient's quality of 
life was the most important factor in the decision to euthanase their pet (Freeman and others 2005). Also, veterinarians strive to improve quality of life in their patients (Yeates and Main 2009). Recent studies have evaluated owners' perception of quality of life in patients receiving lymphoma chemotherapy (Mellanby and others 2003, Tzannes and others 2008) which showed that owners generally felt positive about lymphoma chemotherapy. A questionnaire study was also recently published evaluating owners' perception of medical treatment of cancer showing that owners generally were positive regarding medical treatment of cancer (Bronden and others 2003). Owners' perception of patient quality of life has not been established in patients receiving intravenous carboplatin chemotherapy. The aim of this study was to evaluate owners' perception of quality of life in patients receiving carboplatin in combination with other medications for palliative treatment of a variety of neoplasms. The assessment of the patient's quality of life was made by the owners in a retrospective manner, although the study did include some patients currently receiving carboplatin.

\section{MATERIALS AND METHODS}

This study was conducted in accordance with the Unitec Research Ethics Committee guidelines.

The patient database from the medicine department at the Veterinary Specialist Group (VSG), Auckland, and the patient database at Catmed, Lower Hutt, were reviewed for the period of 2004 to 2009 and all patients receiving one or more doses of intravenous carboplatin were identified. Both centres are private specialist referral practices in New Zealand. Management of cases from both centres was considered to be similar; dogs and cats were included in the study, if they had received one or more doses of carboplatin. Concurrent medications were allowed. Radiation therapy was not performed in either institution during the study period as radiation therapy is not available for pets in New Zealand. All patients were client owned animals and each owner gave informed consent to administer carboplatin before administration.

Information regarding the patient's species, breed, sex, age, presenting signs, diagnosis, the treatment protocol, number of cycles of treatment, treatment delays, concurrent medications and reported side effects were recorded from the case records.

Owners were contacted via a postal questionnaire and were asked a series of questions (Appendix) to evaluate the patient's quality of life before cancer, after diagnosis of cancer, but before treatment and during treatment with carboplatin. The owners were asked to rate their pet's quality of life according to a 10-point numerical rating system with one being "quality of life could not be worse”, and 10 being "quality of life could not be better". Scores were treated as continuous and were able to be marked anywhere along the scale (see Appendix). However, all scores marked on the scales by the participants were either whole numbers or exactly between two numbers. Owners were also asked to evaluate their perception of chemotherapy in pets both before and after treating their pet. Assessment of the patient's side effects was rated as mild, moderate or severe. Criteria were not detailed for any severity level and owners could choose whichever category they felt appropriately classified the clinical signs. Final questions evaluated owners' satisfaction with the treatment according to their pet's quality of life and whether they regretted their decision to treat with carboplatin. Respondents were not questioned as to the average quality of life of their pet during the treatment protocol. Respondents answered anonymously. Owners were given an option not to complete the questionnaire and to withdraw from the study up to 2 weeks after completion of the study period. Non-respondents were not contacted again as non-return of the questionnaire was taken as unwillingness to participate in the study. Data analysis was performed using SPSS $17 \cdot 0$. Differences in quality of life scores were evaluated using a non-parametric Wilcoxon signed-rank test due to the small sample size and moderate skewness of the data. Since there were six independent hypothesis tests being examined, a Bonferroni correction was used to adjust the level of significance from the standard 0.05 to $0.008(0.05$ of 6$)$.

\section{RESULTS}

Forty-seven patients met the study inclusion criteria. Twenty-eight (59\%) owners completed the questionnaire. Twenty-seven patients were treated at VSG and one patient at Catmed clinic. Twenty- 
three dogs and five cats were included in the study. The mean duration from initiation of carboplatin treatment to questionnaire was $22 \cdot 7$ months (range 1 to 51 months).

Twenty-six patients (22 canine and 4 feline patients) received other medications during the time they were treated with carboplatin. Seventeen dogs (74\%) and two cats (40\%) received non-steroidal antiinflammatory medications (the type chosen at the clinician's discretion). Seven dogs (30\%) received doxorubicin. Other medications received by both dogs and cats included metoclopramide (seven), codeine or tramadol (five), bisphosphonate (four), potentiated amoxicillin (four) and mirtazapine (three).

Twelve canine breeds were represented. Of the canine patients, 13 patients were neutered females, 9 were neutered males and 1 an entire male. The median age of dogs at presentation was 11 years (range 5 to 16 years). Seven tumour types were represented; osteosarcoma (seven cases), nasal carcinoma (six cases), oral malignant melanoma (three cases), thyroid carcinoma (two cases) and one each of tonsillar squamous cell carcinoma, anal gland adenocarcinoma and hepatocellular carcinoma. One patient had a solitary pulmonary mass which was not biopsied.

The 23 patients had 99 carboplatin doses. The median number of doses was three per patient (range 1 to 12 doses). The median dose was $300 \mathrm{mg} / \mathrm{m}^{2}$ (range 240 to $300 \mathrm{mg} / \mathrm{m}^{2}$ ). There were three dose reductions due to side effects (details were not specified in the records). Sixteen of 23 dogs had clinical signs related to their tumour before treatment with carboplatin. Nine of these patients had an improvement in their clinical signs.

Of the five feline patients, three were domestic shorthair cats and two were Birmans. Two feline patients were neutered females and three were neutered males. The median age of cats at presentation was 12 years (range 5 to 18 years). Two feline patients had poorly differentiated metastatic carcinoma and the remaining three had one each of renal carcinoma, pancreatic carcinoma and thymoma. Five patients had 17 doses of carboplatin. The median number of doses was two per patient (range two to six doses). The median dose was $200 \mathrm{mg} / \mathrm{m}^{2}$ (range 180 to $210 \mathrm{mg} / \mathrm{m}^{2}$ ) with one dose reduction during treatment due to neutropenia and one dose reduction at initiation of treatment due to presenting azotemia. Three of the five feline patients had clinical signs related to their tumour before treatment with carboplatin and one patient experienced an improvement.

Before their pet's treatment, 12 (43\%) owners had not thought about the use of chemotherapy in animals. Nine (32\%) supported the use of chemotherapy, six (21\%) were equivocal and only one (4\%) owner disagreed with the use of chemotherapy in animals. After treatment, 24 (89\%) owners supported the use of chemotherapy in animals, including the owner who initially disagreed with its use. No owners disagreed with the use of chemotherapy in animals after treating their pet; however, three owners were equivocal regarding the use of chemotherapy in companion animal medicine. One owner did not answer this question.

When asked whether they would treat another pet with carboplatin in the unlikely event this was required, 19 (68\%) owners answered in the affirmative. Eight $(28 \%)$ owners were undecided and one owner said they would not treat another pet with carboplatin. This owner also regretted treating their pet with carboplatin. Sixteen (57\%) owners said they would treat with a different form of chemotherapy in the unlikely event another pet required chemotherapy. Nine owners were undecided, citing it would depend on the diagnosis and prognosis, while three said they would not pursue other chemotherapy options in their pets. One of these owners regretted treating their pet with carboplatin, the other two did not.

Sixteen (57\%) patients experienced side effects during their carboplatin treatment protocols (13 dogs and 3 cats). Side effects were considered by the owner to be mild (six cases), mild to moderate (one case), moderate (two cases) and moderate to severe (three cases). Side effects included tiredness and lethargy (15), loss of appetite (7), vomiting (3), diarrhoea (2) and hematochezia (1). Tiredness/ lethargy was considered mild (10), moderate (3), mild to moderate (1) and severe (1). Loss of appetite was considered mild (three), moderate (one), mild to moderate (one) and severe (two). Vomiting was considered mild, moderate and severe with one case in each category. Diarrhoea was considered mild 
to moderate in one case and severe in one case. The patient with severe diarrhoea also had hematochezia reported. Only one patient with mild side effects required treatment (for loss of appetite). Patients with moderate to severe side effects required treatment for a variety of side effects (including loss of appetite (four), lethargy (two), diarrhoea (two), vomiting (one) and hematochezia (one)).

Table 1 shows the descriptive statistics for the quality of life scores at the various stages of illness. The mean and median quality of life scores are presented in the table, but the analysis focusses on the median differences due to the skewness of median quality of life score before the diagnosis was 10 . Twenty-five owners reported that their pet's quality of life decreased, resulting in a significantly lower quality of life score $(\mathrm{P}<0 \cdot 001)$ once the diagnosis of cancer was made but before treatment was initiated (Table 2). The median decrease in the quality of life during treatment was also significant relative to precancer levels; at its best (median=8, $\mathrm{P}<0 \cdot 001$ ), and at its worst (median=5, $\mathrm{P}<0 \cdot 001$ ). At its best, the median difference in the quality of life scores was significant, indicating higher scores for most patients during treatment compared to before treatment (but postdiagnosis) $(\mathrm{P}=0 \cdot 005)$. Eighteen patients had an increase in quality of life scores, five remained the same and only four patients experienced a decrease in their quality of life scores at its best during the treatment compared with pretreatment (but postdiagnosis) quality of life. At its worst, however, the median quality of life scores was not significantly different when compared to the pretreatment (but postdiagnosis) scores $(\mathrm{P}=0 \cdot 044)$. At its best during treatment (median=8), the quality of life scores were significantly higher than at its worst (median=5) $(\mathrm{P}<0 \cdot 001)$.

\begin{tabular}{lccccc}
$\begin{array}{l}\text { Table } \mathbf{1} \text { Q Quality of life scores, rated on a scale of } \mathbf{1} \text { (could not be worse) to } \mathbf{1 0} \\
\text { (could not be better) }\end{array}$ & $\mathbf{n}$ & Mean (sd) & Median & Min, Max & LQ, UQ \\
\hline Quality of life score & 28 & $9 \cdot 3(0.9)$ & 10 & 7,10 & 9,10 \\
Before cancer & 28 & $6 \cdot 4(2.2)$ & 7 & 2,10 & 4,8 \\
After diagnosis but before treatment & 27 & $7 \cdot 9(1.9)$ & 8 & 3,10 & 7,9 \\
At its best during treatment & 27 & $5 \cdot 3(2.4)$ & 5 & $1,9.5$ & $4,7 \cdot 5$ \\
At its worst during treatment & 27
\end{tabular}

SD Standard deviation

LQ Lower quartile (cuts off the lowest $25 \%$ of the data)

UQ Upper quartile (cuts off the highest $25 \%$ of the data)

Table 2. Differences betweeen quality of life scores using a Wilcoxon signedrank test

Comparison of quality of life score

Before cancer and after diagnosis but before treatment

Before cancer and at its best during treatment

Before cancer and at its worst during treatment

Before treatment and at its best during treatment

Before treatment and at its worst during treatment

At its best and worst during treatment
Quality of life: number of patients with an increase/same/

\section{decrease}

$0 / 3 / 25$

$<0 \cdot 001$

$0 / 10 / 17$

$<0.001$

$0 / 1 / 26$

$<0.001$

$18 / 5 / 4$

0.005

$8 / 3 / 16$

0.043

$0 / 6 / 21$ 
Eighteen (64\%) owners stated that the cost of treatment did not matter. Eight owners found the cost acceptable for the benefit. Two respondents chose option "other" and wrote individual comments rather than choosing a suggested comment. One owner felt the "cost was not acceptable for the time it gave". This patient had severe side effects, no improvement in its clinical signs and the owners regretted the treatment. The remaining owner commented that the treatment was "too expensive but the extra time was great”.

Twenty-three (82\%) owners reported treating with carboplatin did not alter their relationship with their pet. One owner reported that their relationship deteriorated but was still acceptable. This patient had mild to moderate side effects and the owner would treat with carboplatin again if required however regretted treatment in this pet. Four owners reported that their relationship improved. Three of these patients had mild side effects.

Owners were asked whether they regretted treating their pet with carboplatin. Twenty-five (89\%) owners did not regret their decision to treat. Three owners regretted treatment.

\section{DISCUSSION}

This study showed that the majority of owners felt that their experience with carboplatin in conjunction with other palliative treatments was positive and that most owners would treat another pet with carboplatin if it were required. More than half the patients had side effects during their treatment protocol and only 58\% of pets had an improvement in their initial presenting signs. Even so, treating with carboplatin changed most owners' ideas about chemotherapy in pets from negative or neutral to positive. This positive experience parallels the results of other veterinary oncology quality of life studies (Mellanby and others 2003, Bronden and others 2003, Tzannes and other 2008). Interestingly, one respondent was adverse to chemotherapy in pets before proceeding with carboplatin treatment. The reason behind this is not clear due to the anonymity of the questionnaire, which was a requirement of the study's ethics committee. However, it may be that the respondent filling in the questionnaire was not the owner who made the initial decision to proceed with treatment and that their opinion subsequently changed with treatment, or it may be that their reasoning behind being adverse to treatment was based on misconceptions about the treatment (for example, side effects, quality of life concerns, costs of treatment in general or carboplatin in particular and so on) and once able to make an informed decision their perception of chemotherapy in pets changed.

Although most owners felt that their experience with carboplatin treatment was positive, $11 \%$ (three owners) regretted treatment. Owners were not asked in the questionnaire their reasons for regretting treatment; however, these reasons could be multi-factorial and include a lack of response to treatment, unrealistic expectations, side effects of the medication(s), a decrease in quality of life for their pet during treatment or financial or emotional costs of palliative treatment. Also, some pets experienced owner rated poor quality of life during the time they received carboplatin. This could have been due to side effects of the treatments they received, or due to progression of an underlying disease process, or a combination of the two.

Owners were more likely to retrospectively rate the carboplatin treatment as negative if their pet had no improvement in their clinical signs. They were also more likely to rate treatment as negative if their pet experienced side effects during treatment. It is possible that owners had difficulty separating the clinical signs associated with the underlying disease process from side effects associated with treatment, and therefore may have overestimated the number or severity of the side effects their pets experienced. Conversely, it is also possible that owners underestimated the severity of clinical signs in their pets. This may have been due to a variety of reasons including a desire to remember only positive experiences with their pet or recall bias due to the length of time between treatment and questionnaire. Even though patients had reduced quality of life scores during treatment compared with their precancer diagnosis quality of life, at its best, treatment did improve the quality of life significantly from pretreatment quality of life. At its worst, there was a non-significant trend towards worse quality of life compared with pretreatment quality of life scores. This is encouraging information for both clinicians and clients alike. Despite a decrease in quality of life compared to prediagnosis, only three owners regretted treatment. Tolerance to a decrease in quality of life has also previously been reported in 
owners whose cats were receiving chemotherapy for lymphoma (Tzannes and others 2008). This may reflect a more motivated population of owners who are more willing to tolerate a reduction in quality of life for a perceived or hoped increased quantity of life (Tzannes and others 2008).

A great deal of variation was noted in patient quality of life during the time they received carboplatin. The median decrease in the quality of life scores from at their best during treatment (median=8) to at their worst (median=5) was signifi cantly greater than zero $(\mathrm{P}<0 \cdot 001)$. There was also a variation in quality of life between patients. At its best, quality of life scores varied from 3 to 10 whereas at its worst, quality of life scores varied from 1 to $9 \cdot 5$. This inter-individual variation could represent the variation in either the underlying disease process between patients or side effects of the treatment regimes, with some patients experiencing owner rated severe side effects while some experienced no side effects. It was also possible that there was poor inter-observer reliability, as each owner could have a different perception or understanding of quality of life, different interpretations of the question and use of the grading scale.

Most owners did not consider the cost of treatment to be a factor in the decision making process, or they felt that the cost of treatment was worth the perceived benefit. It is possible that this reflects a referral population who are potentially more willing or able to spend money on their pets; however, in a recent study, $70 \cdot 8 \%$ of responding United Kingdom veterinary practices prescribed cytotoxic drugs, with the majority of these being primary care facilities (Cave and others 2007). It may be that pets are being acknowledged more as a family member than purely as an animal and that this may influence owners' willingness to invest in a treatment for their pets that is considered palliative (Shaw and Lagoni 2007).

Almost all patients included in the study received concurrent medications. Ideally, for a study on quality of life for a certain medication, the medication of interest would be the sole medication the patient received during the study period. However, given the palliative nature of carboplatin chemotherapy, patients are frequently prescribed multiple medications. Therefore, although not ideal from a research perspective, the results of the study do mirror the situation in clinical practice and therefore are potentially more relevant to clinicians and owners as it aligns more closely with what they will be experiencing. Given the number of concurrent medications, it is possible that respondents were attributing patient side effects to carboplatin when they were actually a result of one (or more) of the other medications they were receiving; thus overestimating the side effects seen with carboplatin use. Conversely, it is possible that they attributed the side effects of carboplatin to one of the other medications; therefore, resulting in an underestimation of actual side effects. Thirty per cent of dogs received doxorubicin before, during or after their treatment with carboplatin, which may have resulted in erroneous attribution of side effects to carboplatin (overestimating side effects) or to doxorubicin (underestimating side effects). Limitations notwithstanding, the results are still valuable to clinicians and owners as this is the way carboplatin is often used in practice.

All but one owner felt that their relationship with their pet was either improved or the same as before treatment with carboplatin. The owner's relationship, or human-companion animal bond is often paramount to owners and the thought of an intervention adversely affecting this bond or relationship is worrying to them (Friedmann and Son 2009). One owner commented that their relationship had improved with their pet because "they appreciated her more" as they "admired her strength and positive nature”. Only one owner felt that their relationship with their pet had deteriorated; this pet had mild to moderate side effects. Even though many of the patients had side effects, some of which were categorised as severe by the owners, the majority of owners did not feel that this adversely affected their relationship with their pet.

Fifty-nine per cent of questionnaires were returned and this may have resulted in a sampling bias. Only one questionnaire was returned from the Catmed clinic. As the philosophy of treatment and treatment regimes were very similar at both centres, the authors chose not to exclude the questionnaire results from the study. Demographic information related to owners was not asked in the questionnaire to allow respondents' to remain anonymous; therefore, comparisons of owners to see if the respondents were representative of the entire sampling population was not possible. Owners who were positive about the experience may have been more likely to respond than owners who had a negative or adverse experience (Edwards and others 2007). However, there were a number of side effects reported and a 
number of owners who regretted treatment. This suggests that the respondent's may well be representative of the sample population and therefore results may be a valid indicator of owner's perception of their pet's quality of life during carboplatin treatment. Also, most pets had side effects; however, most owners did not regret their decision to treat.

There are no standardised quality of life instruments in veterinary medicine for evaluation of chemotherapy protocols (Wojciechowska and Hewson 2005). Human quality of life instruments for proxy informants has been adapted in numerous veterinary papers (Freeman and others 2003, Mellanby and others 2003, Tzannes and others 2008). A number of studies have been performed assessing quality of life in pets using a Likert scale or numerical rating scale to assess the owner's perception of quality of life. In human studies, proxy informants have been shown to both underestimate and overestimate quality of life (Yazbec and Fantoni 2005, Jozefiak and others 2008). This has not been studied in veterinary medicine as it is impossible to comment on quality of life from the animal's point of view (as all assessments are still human rated parameters); however, this effect may exist (Yeates and Main 2009) and therefore it may be that owners as proxy informants also underestimate or overestimate their pet's quality of life.

Specific criteria were not given to denote what were classified as mild, moderate or severe side effects. Medically, side effects can be very specifically graded (for example grade I diarrhoea is classified as soft formed faeces while grade II diarrhoea is classified as less than seven watery stools for less than 7 days (Cave 2006)). One owner may feel that the presence of diarrhoea was enough to classify it as severe while another owner may classify medically severe diarrhoea as mild or moderate. Criteria such as this were not set as the question regarding the owners perception of their pet's side effects was to obtain just that, their perception.

Quantification of quality of life using a numerical rating scale is not standardised as each individual will have their own scale with which to judge the response (Yeates and Main 2009). A score of 10 in one pet may be the equivalent of a seven in another. The question regarding patient's quality of life before the development of cancer was asked in an effort to account for individual differences with the numerical rating scale (Mellanby and others 2003). The numerical rating scale was chosen as it potentially provided a more sensitive index of patient's quality of life than choosing between three or four categories (Mellanby and others 2003). A 1- to 10-point scale was chosen to minimise potential over-inflation of quality of life that may have occurred if the lowest point was zero. Owners may have been less willing to use numbers closer to or including zero if they felt they would be judged for consenting to a treatment which afforded no quality of life (at best) or possibly suggesting the patient died due to the side effects of the treatment regime.

The questions included in this paper were subjective in nature. A previous paper assessing quality of life in pets with cancer pain suggested that subjective questions may not allow the true condition of an animal to be assessed (Yazbec and Fantoni 2005). Subjective questions were chosen as the owners were asked the questions in a retrospective manner and were potentially less likely to remember specifics of their pet's behaviour and demean-our. Some patients received carboplatin up to 5 years previously and therefore there may be a degree of recall bias in the results (Tzannes and others 2008). It is possible that some patients may have had side effects recorded in their records but not recalled by the owners in the questionnaire, however, as the owners answered the questionnaires anonymously, this information is not available.

The results of this study may be complicated by recall bias. As the study was retrospective in nature, many respondents were answering questions about events which happened up to five years previously. A study in human medicine conducted over a four year period showed recall bias with some facts (such as the brand of medication) but not others (such as length of use of medication) (Nischan and others 1993). It is also possible that any recall bias was further complicated by the proxy informant classification of the respondents.

A specific limitation of this study is that a question was not asked regarding average quality of life during treatment. The absence of this data means that we cannot comment on whether owners perceived quality of life to be generally improved or worse than pretreatment (postdiagnosis) quality of life. This information would be interesting and beneficial to both owners and clinicians and further 
studies would be useful to evaluate this information. Also, further evaluation into the percentage time the owners felt they experienced good or bad quality of life would be beneficial.

The response rate was lower in this study than a previously published veterinary oncology survey (Tzannes and others 2008), but is similar to previously published human medical surveys (Bergk and others 2005). Many factors have been reported to be associated with increased or decreased response and it may be that the reason for this is multi-factorial (Edwards and others 2007). The sample size was small reducing the ability to obtain subgroup comparisons. There were insufficient numbers to allow statistical assessment of owners' feelings regarding treatment and regrets compared with the occurrence of clinical signs.

Despite the limitations, this study found that carboplatin treatment was well tolerated by both owners and patients, many patients had an improvement in their cancer-related clinical signs and for at least some of the time during treatment patients had an improvement in their quality of life compared with that before treatment. Most owners had no regrets regarding treatment. These findings indicate that veterinarians should feel confident offering carboplatin alone or in conjunction with other medications (such as non-steroidal anti-inflammatories, doxorubicin, potentiated amoxicillin and metoclopramide) as a palliative treatment for susceptible neoplasia in dogs and cats.

\section{Acknowledgement}

This project was funded by a Companion Animal Society - New Zealand Companion Animal Health Fund Annual Study Grant.

\section{References}

BENNETT, P. F., DENICOLA, D. B., BONNEY, P.,GLICKMAN, N.W. \& KNAPP, D. W. (2002) Canine anal sac adenocarcinomas: clinical presentation and response to therapy. Journal of Veterinary Internal Medicine 16, 100-104

BERGK, V., GASSE, C., SCHNELL, R., \& HAEFELI, W. E. (2005) Mail surveys: obsolescent model or valuable instrument in general practice. Swiss Medical Weekly 135, 189-191

BERGMAN, P. J., MACEWEN, G., KURZMAN, I. D., HENRY, C. J., HAMMER, A. S., KNAPP, D. W., HALE, A., KRUTH, S. A., KLEIN, M. K., KLAUSNER, J., NORRIS, A. M., MCCAW, D., STRAW, R. C. \& WITHROW, S. J. (1996) Amputation and carboplatin for treatment of dogs with osteosarcoma: 48 cases (1991 to 1993). Journal of Veterinary Internal Medicine 10, 76-81

BRONDEN, L. B., RUTTEMAN, G. R., FLAGSTAD, A. \& TESKE, E. (2003) Study of dog and cat owners' perception of medical treatment for cancer. Veterinary Record 152, 77-80

CAVE, T. (2006) Risk factors for diarrhoea during chemotherapy with cyclophosphamide, epirubicin, vincristine and prednisolone for canine lymphoma. Proceedings of the British Small Animal Veterinary Congress. April 20 to 23, Birmingham, UK. http:// www.vin.com [accessed 23 August 2009]

CAVE, T. A., NORMAN, P. \& MELLOR, D. (2007) Cytotoxic drug use in treatment of dogs and cats with cancer by UK veterinary practices (2003 to 2004). Journal of Small Animal Practice 48, 371-377

DHALIWAL, R. S., JOHNSON, T. O. \& KITCHELL, B. E. (2003) Primary extraskeletal hepatic osteosarcoma in a cat. Journal of the Veterinary Medical Association 222, 340-342

DOMINQUEZ, P. A., DERVISIS, N. G., CADILE, C. D., SARBU, L. \& KITCHEN, B. E. (2009) Combined gemcitabine and carboplatin therapy for carcinomas in dogs. Journal of Veterinary Internal Medicine 23, 130-137

EDWARDS, P., ROBERTS, I., CLARKE, M., DEGUISEPPI, C., PRATAP, S., WENTZ, R., KWAN, I. \& COOPER, R. (2007) Methods to increase response rates to postal questionnaires. Cochrane Database Systemic Review 18, MR00008

FOX, L. E. (2000) Carboplatin. Journal of the American Animal Hospital Association 36, 13-14

FREEMAN, K. P., HAHN, K. A., HARRIS, F. D. \& KING, G. K. (2003) Treatment of dogs with oral melanoma by hypofractionated radiation therapy and platinum-based chemotherapy (1987-1997). Journal of Veterinary Internal Medicine 17, 96-101

FREEMAN, L. M., RUSH, J. E. \& FARABAUGH, A. E. (2005) Development and evaluation of a questionnaire for assessing health related quality of life in dogs with cardiac disease. Journal of the American Veterinary Medical Association 226, 1864-1868

FRIEDMANN, E. \& SON, H. (2009) The human-companion animal bond: how humans benefit. Veterinary Clinics of North America: Small Animal Practice 39, 293-326 
HAHN, H. A., MCENTEE, M. F., DANIEL, G. B., LEGENDRE, A. M. \& NOLAN, M. L. (1997) Hematologic and systemic toxicoses associated with carboplatin administration in cats. American Journal of Veterinary Research 58, 677-679

JOZEFIAK, T., LARSSON, B., WICHSTROM, L., MATTEJAT, F. \& RAVENS-SIEBERER, U. (2008) Quality of life as reported by school children and their parents: a cross sectional survey. Health and Quality of Life Outcomes 6, 34

KISSEBERTH, W. C., VAIL, D. M., YAISSLE, J., JEGLUM, K. A., COUTO, C. G., WARD, H., KHANNA, C. \& OBRADOVICH, J.

E. (2008) Phase I clinical evaluation of carboplatin in tumor-bearing cats: A Veterinary Cooperative Oncology Group study. Journal of Veterinary Internal Medicine 22, 83-88

LANGOVA, V., MUTSAUERS, A. J., PHILLIPS, B. \& STRAW, R. (2004) Treatment of eight dogs with nasal tumours with alternating doses of doxorubicin and carboplatin in conjunction with oral piroxicam. Australian Veterinary Journal 82, 676-680

MELLANBY R. J., HERRTAGE, M. E. \& DOBSON, J. M. (2003) Owners' assessments of their dog's quality of life during palliative chemotherapy for lymphoma. Journal of Small Animal Practice 44, 100-103

MURPHY, S., HAYES, A., ADAMS, V., MAGLENNON, G., NEATH, P., LADLOW, J. \& BREARLEY, M. J. (2006) Role of carboplatin in multimodal treatment of canine tonsillar squamous cell carcinoma - a case series of fi ve dogs. Journal of Small Animal Practice 47, 216220

NISCHAN, P., EBELING, K., THOMAS, D. B. \& HIRSCH, U. (1993) Comparison of recalled and validated oral contraceptive histories. American Journal of Epidemiology 138, 697-703

OSOBA, D. (1992) The quality of life committee of the clinical trials group of the national cancer institute of Canada: organisations and functions. Quality of Life Research 1, 211-218

PAGE, R. L., MCENTEE, M. C., GEORGE, S. L., WILLIAMS, P. L., HEIDNER, G. L., NOVOTNEY, C. A., RIVIERE, J. E., DEWHIRST, M. W. \& THRALL, D. E. (1993) Pharmacokinetic and phase I evaluation of carboplatin in dogs. Journal of Veterinary Internal Medicine 7, 235-240

SHAW, J. R. \& LAGONI, L. (2007) End-of-life communication in veterinary medicine: delivering bad news and euthanasia decision making. Veterinary Clinics of North America Small Animal Practice 37, 95-108

SPUGNINI, E. P., RUSLANDER, D. \& BARTOLAZZI, A. (2001) Extraskeletal osteosarcoma in a cat. Journal of the Veterinary Medical Association 219, 60-62

SPUGNINI, E. P., CRISPI, S., SCARABELLO, A., CARUSO, G., CITRO, G. \& BALDI, A. (2008) Piroxicam and intracavitary platinum-based chemotherapy for the treatment of advanced mesothelioma in pets: preliminary observations. Journal of Experimental and Clinical Cancer Research 27, 6

THEON, A. P., VANVECHTEN, M. K. \& MADEWELL, B. R. (1996) Intratumoral administration of carboplatin for treatment of squamous cell carcinomas of the nasal plane in cats. American Journal of Veterinary Research 57, 205-210

TZANNES, S., HAMMOND, M. F., MURPHY, S., SPARKES, A. \& BLACKWOOD, L. (2008) Owners 'perception of their cats' quality of life during COP chemotherapy for lymphoma. Journal of Feline Medicine and Surgery 10, 73-81

WOJCIECHOWSKA, J. I. \& HEWSON, C. J. (2005) Quality of life assessment in pet dogs. Journal of the American Veterinary Medical Association 226, 722-728

YAZBEC, K. V. B. \& FANTONI, D. T. (2005) Validity of a health related quality of life scale for dogs with signs of pain secondary to cancer. Journal of the American Veterinary Medical Association 226, 1354-1358

YEATES, J. \& MAIN, D. (2009) Assessment of companion animal quality of life in veterinary practice and research. Journal of Small Animal Practice 50, 274-281 


\section{APPENDIX}

1. Is/was your pet a:

$$
\square \text { Dog }
$$

$\square$ Cat

2. Prior to treating your pet with carboplatin, which of these comments best describes your feelings about chemotherapy in pets?

I supported the use of chemotherapy in pets

I disagreed with the use of chemotherapy in pets

I had no strong feelings either way

$\square \quad 1$ had not thought about the use of chemotherapy in pets

3. After treating your pet with carboplatin, which of these comments best describes your feelings about chemotherapy in pets?

I support the use of chemotherapy in pets

I disagree with the use of chemotherapy in pets

I have no strong feelings either way

4. Did your pet have any clinical signs (symptoms) related to their cancer prior to treatment?

$\square$ Yes - please answer question 5

No-please skip question 5 and proceed to question 6

5. Did your pet's elinical signs (symptoms) improve with treatment with carboplatin?

$\square$ Yes

$\square$ No

6. Did your pet experience any side effects of carboplatin treatment?

$\square \quad$ Yes - please answer question 7

$\square$ No-please proceed to question 12

7. What were your pet's main side effects?
Tiredness/lethargy
Loss of appetite
Vomiting
Diarrhoea
Other (please specify)

8. How would you rate your pet's side effects?
$\square$ Mild
$\square$ Moderate
$\square$ Severe

9. Did the side effects your pet experienced require treatment at a veterinary clinic?

$\square$ No-please proceed to question 12

$\square$ Yes - please answer question 10

10. What were the side effects that prompted veterinary treatment?
$\square$ Tiredness/lethargy
$\square$ Loss of appetite
Vomiting
$\square$ Diarrhoea
$\square$ Other (please specify)

11. Did your pet get this treatment at the veterinary clinic which administered the carboplatin?

$\square$ Yes

$\square \quad$ No

12. What was your pet's quality of life before the symptoms of cancer were apparent?

\begin{tabular}{llllllllll}
\hline 1 & 2 & 3 & 4 & 5 & 6 & 7 & 8 & 9 & 10 \\
\hline
\end{tabular}

Quality of life

could not be

Quality of life

worse

could not

13. What was your pet's quality of life once the cancer had been diagnosed but before treatment with carboplatin?

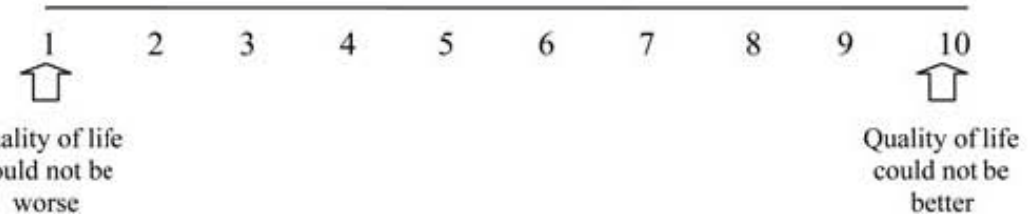




\begin{tabular}{|c|c|c|c|c|c|c|c|}
\hline 1 & 3 & 4 & 5 & 6 & 7 & 8 & 10 \\
\hline $\begin{array}{l}\text { Quality of life } \\
\text { could not be } \\
\text { worse }\end{array}$ & & & & & & & $\begin{array}{l}\text { Quality of life } \\
\text { could not be } \\
\text { better }\end{array}$ \\
\hline
\end{tabular}

13. What was your pet's quality of life once the cancer had been diagnosed but before treatment with carboplatin?

$\int^{1}$
$\begin{gathered}\text { Quality of life } \\ \text { could not be } \\ \text { worse }\end{gathered}$

14. When your pet's quality of life is/was at its BEST during treatment with carboplatin What is/was your pet's quality of life?

$\begin{array}{llllllllll}1 & 2 & 3 & 4 & 5 & 6 & 7 & 8 & 9 & \underbrace{10}_{\begin{array}{c}\text { Quality of life } \\ \text { could not be } \\ \text { better }\end{array}} \\ \begin{array}{c}\text { could not be } \\ \text { worse }\end{array} & & & & & & & & & \end{array}$

15. When your pet's quality of life was at its WORST during treatment with carboplatin What was/is your pet's quality of life?

\begin{tabular}{|c|c|c|c|c|c|c|c|}
\hline 1 & 3 & 4 & 5 & 6 & 7 & 8 & 9 \\
\hline $\begin{array}{l}\text { tality of life } \\
\text { puld not be } \\
\text { worse }\end{array}$ & & & & & & & \\
\hline
\end{tabular}

16. Has treating your pet with carboplatin affected your relationship with your pet?

$\square$ Quality of relationship has improved since beginning treatment with carboplatin

$\square$ Quality of relationship is the same as prior to my pet beginning treatment

$\square$ Quality of relationship deteriorated but still acceptable

$\square$ Quality of relationship deteriorated and unacceptable

17. Would you undertake carboplatin chemotherapy again in the future in the unlikely event another pet required carboplatin treatment?
$\square$ Yes
$\square$ No
$\square$ Undecided

18. Would you undertake different chemotherapy treatment in a pet if in the unlikely event another pet required a different type of chemotherapy?
$\square$ Yes
$\square$ No
$\square$ Undecided

19. How do you feel about the cost of treatment?
$\square$ Cost did not matter
Cost was acceptable for the time it gave us
$\square$ Cost was unacceptable for the time it gave us
$\square \quad$ Other (please specify)

20. Do you regret treating your pet with carboplatin?
$\square$ Yes
$\square$ No
$\square$ Undecided 